12,03

\title{
Электронная структура порфирина никеля NiP: исследование методами рентгеновской фотоэлектронной и абсорбционной спектроскопии
}

\author{
() Г.И. Свирский ${ }^{1}$, Н.Н. Сергеева ${ }^{2}$, С.А. Красников ${ }^{1,3}$, Н.А. Виноградов ${ }^{1,4}$, Ю.Н. Сергеева ${ }^{5}$, \\ A.A. Cafolla ${ }^{3}$, A.Б. Преображенский ${ }^{1,4}$, A.C. Виноградов ${ }^{1,9}$ \\ ${ }^{1}$ Санкт-Петербургский государственный университет, \\ Санкт-Петербург, Россия \\ ${ }^{2}$ School of Chemistry, University of Leeds, \\ Leeds, U.K. \\ ${ }^{3}$ School of Physical Sciences, Dublin City University, \\ Glasnevin, Dublin, Republic of Ireland \\ ${ }^{4}$ MAX IV Laboratory, University of Lund, \\ Lund, Sweden \\ ${ }^{5}$ Commissariat à l'énergie atomique et aux énergies alternatives, Institut Nanosciences et Cryogénie, \\ Grenoble, France \\ ฯ E-mail: asvinograd@gmail.com
}

(Поступила в Редакцию 22 июня 2016 г.)

\begin{abstract}
Энергетические распределения и свойства занятых и свободных электронных состояний для плоского комплекса порфирина никеля NiP исследованы методами рентгеновской фотоэмиссионной и абсорбционной спектроскопии. В результате анализа экспериментальных спектров валентной фотоэмиссии определен характер и энергетические положения верхних занятых электронных состояний: высшее занятое состояние сформировано в основном атомными состояниями порфинового лиганда, следующие два состояния связаны с $3 d$-состояниями атома никеля. Установлено, что низшее свободное состояние имеет специфический характер и описывается $b_{1 g}$-MO $\sigma$-типа, которая формируется свободными $\mathrm{Ni} 3 d_{x^{2}-y^{2}}$-состояниями и занятыми $2 p$-состояниями неподеленных электронных пар атомов азота. Этот специфический характер низшего свободного состояния является следствием донорно-акцепторной химической связи в NiP.
\end{abstract}

Работа выполнена частично в рамках двухсторонней Программы „Российско-Германская лаборатория БЭССИ“ при поддержке Российского фонда фундаментальных исследований (грант № 15-02-06369).

DOI: $10.21883 /$ FTT.2017.02.44063.255

\section{1. Введение}

Современный интерес к молекулярным комплексам металлопорфиринов (MPs) обусловлен их ключевой ролью в различных биохимических процессах и широким использованием во множестве технологических приложений [1]. Порфирины переходных $3 d$-металлов (3d-MPs) - среди наиболее популярных кандидатов на широкое использование в молекулярной электронике [2], нелинейной оптике [3], катализе [4], диагностике и терапии рака [5], сенсорике [6] и многих других направлениях современных технологий. Одним из наиболее простых порфиринов $3 d$-металлов является порфин никеля(II) NiP, электронное строение которого, согласно оптическим экспериментами $[7,8]$ и квантовохимическим расчетам $[9,10]$, характеризуется полностью заполненными электронными оболочками. Вследствие этого, молекула NiP имеет в основном состоянии суммарный спин, равный нулю, что заметно упрощает анализ и интерпретацию спектроскопических данных. Следует также заметить, что молекула NiP является прародителем большого семейства производных порфиринов никеля, которые получаются присоединением к
NiP на его периферии разнообразных функциональных групп. Подобная химическая функционализация простейшего порфирина никеля существенно расширяет спектр полезных свойств его семейства. В связи с вышеуказанным очевидно, что всесторонние экспериментальные и теоретические исследования атомного и электронного строения $\mathrm{NiP}$ очень важны для понимания свойств этого комплекса и, в сущности, представляют необходимый первый шаг в изучении электронных свойств производных порфиринов никеля, а также более сложных порфиринов $3 d$-металлов с частично заполненными электронными оболочками. Особое место в этих исследованиях должно отводиться экспериментальному изучению полного спектра занятых и свободных электронных состояний $\mathrm{NiP}$, который определяет все физикохимические свойства изучаемого соединения.

Экспериментальные исследования свойств электронной подсистемы порфиринов никеля до сих выполнялись в основном с применением методов оптической спектроскопии поглощения в УФ, видимом и ИК-диапазонах $[11,12]$. Однако подобные эксперименты не обеспечивают получение необходимой информации о занятых и свободных электронных состояниях в порфиринах ни- 
келя, поскольку она содержится в оптических спектрах опосредованно в форме энергий и сил осцилляторов наблюдаемых электронных переходов. Идентификация абсорбционных полос в оптических спектрах комплекса $\mathrm{NiP}$ и его производных затруднена вследствие сложного атомно-орбитального состава молекулярных орбиталей (MO), между которыми происходит электронный переход, и практически невозможна без квантово-химических расчетов электронной структуры комплексов. Кроме того, полосам оптического поглощения соответствуют переходы валентных электронов с энергией в несколько eV между занятыми и свободными состояниями, расположенными только вблизи уровня Ферми.

Рентгеновские спектры поглощения, напротив, формируются переходами электронов из внутренних оболочек атомов, образующих полиатомную систему (молекулу, комплекс). В результате начальное состояние рентгеновского перехода, локализованное на отдельном атоме, описывается атомной орбиталью $(\mathrm{AO})$ с определенной угловой симметрией, что существенно упрощает идентификацию конечного молекулярного состояния (МО) и в целом анализ тонкой структуры рентгеновских спектров поглощения. Метод рентгеновской абсорбционной спектроскопии, основанный на анализе ближней тонкой структуры рентгеновских спектров поглощения БТС РСП (NEXAFS - Near Edge X-ray Absorption Fine Structure) [13], относится к числу наиболее информативных методов и широко используется в настоящее время для изучения полиатомных систем благодаря своей чувствительности к таким деталям локального электронного и атомного строения системы, как порядок следования, энергия связи, атомно-орбитальный состав и угловая симметрия свободных электронных состояний, длины и углы связей, координация ближайшего окружения и др.

В свою очередь, рентгеновская фотоэлектронная спектроскопия является современным и популярным методом зондирования занятых электронных состояний в полиатомных системах [14]. При изучении спектра электронных состояний и характеризации химической связи в соединении, как правило, используются рентгеновская фотоэлектронная спектроскопия внутренних (остовных) электронов (XPS, X-ray photoelectron spectroscopy) и валентных электронов (фотоэмиссия из валентной зоны, VB-PE - valence band photoemission).

Известно несколько работ, в которых XPS-спектры были измерены с невысоким энергетическим разрешением и наряду с оптическими спектрами поглощения использованы для характеризации электронной структуры и химического связывания в порфиринах никеля $[15,16]$. Измерения валентной фотоэмиссии для NiP в литературе отсутствуют. Число рентгеноабсорбционных исследований порфиринов никеля также невелико, и они, как правило, ограничены анализом узкой начальной области спектров поглощения, которая отражает взаимодействие атома никеля с пиррольными атомами азота [17-20].

Основной целью данной работы являлось получение методами рентгеновской фотоэлектронной и абсорбци- онной спектроскопии детальной информации об энергетическом распределении, угловой симметрии, локализации и атомно-орбитальном составе занятых и свободных электронных состояний в молекулярном комплексе порфирина никеля NiP.

\section{2. Экспериментальная часть}

Все измерения были выполнены, используя оборудование Российско-Германского канала вывода и монохроматизации синхротронного излучения (СИ) электронного накопителя БЭССИ II (BESSY II, Berlin, Germany) [21] и канала D1011 вывода и монохроматизации СИ электронного накопителя MAKC-II (Lund, Sweden) [22] при обеспечении близких экспериментальных условий. Порфирин никеля $\mathrm{NiP}$ и свободный (без атома металла) порфин $\mathrm{H}_{2} \mathrm{P}$ были синтезированы с помощью методик, известных из литературы [23]. Образцы для измерений представляли собой поликристаллические слои $\mathrm{NiP}$ толщиной около $30 \mathrm{~nm}$, приготовленные in situ путем термического испарения порошка порфирина из танталового тигля ячейки Кнудсена и осаждения на чистую поверхность медной пластинки. Температура тигля при напылении составляла около $600 \mathrm{~K}$. Скорость напыления контролировалась кварцевым монитором и была около $0.6 \mathrm{~nm} / \mathrm{min}$. Давление паров порфирина в препарационной камере при напылении не превышало $5 \cdot 10^{-8}$ mbar. Образец свободного порфина $\mathrm{H}_{2} \mathrm{P}$ был приготовлен в тех же экспериментальных условиях.

Рентгеновские фотоэлектронные спектры для валентной зоны и остовных уровней $(\mathrm{Ni} 2 p, \mathrm{~N} 1 s$ и $\mathrm{C} 1 s)$ порфирина никеля были измерены с помощью полусферического электронного анализатора Phoibos 150 (Specs $\mathrm{GmbH}$ в в режиме регистрации нормальной интегральной фотоэмиссии. Полное энергетическое разрешение (монохроматора и анализатора) для спектров валентной фотоэмиссии, полученных с использованием возбуждающих квантов с энергией $72-170 \mathrm{eV}$, составляло около $200 \mathrm{meV}$. Спектры остовных уровней возбуждались квантами с энергией 400, 500 и $940 \mathrm{eV}$, при этом полное энергетическое разрешение составляло 300, 350 и $700 \mathrm{meV}$ соответственно. NEXAFS-спектры исследуемых порфиринов были получены методом полного электронного выхода внешнего рентгеновского фотоэффекта в режиме измерения тока утечки с образца при варьировании энергии квантов, падающих на образец под углом $45^{\circ}$. Энергетическое разрешение монохроматора в области Ni2p $3 / 2(\sim 850 \mathrm{eV}), \mathrm{N} 1 s(\sim 400 \mathrm{eV})$ and $\mathrm{C} 1 s(\sim 280 \mathrm{eV})$ краев поглощения составляло 550, 180 и $110 \mathrm{meV}$ соответственно.

Bce спектры фотоэмиссии и поглощения нормировались на падающий поток фотонов, который мониторировался путем регистрации фототока с золотой сетки, установленной на выходе канала вывода СИ. Энергия фотонов в области 70-500 eV калибровалась с помощью фотоэлектронных $\mathrm{Au} 4 f_{7 / 2^{-}}, \mathrm{C} 1 s$ - и $\mathrm{N} 1 s$-спектров, измеренных с излучением, отраженным дифракционной 
решеткой в первом и втором порядках дифракции. Для высокоэнергетической области спектра калибровка осуществлялась с помощью первого узкого пика $(683.9 \mathrm{eV})$ в $\mathrm{F} 1 s$-спектре поглощения твердофазного $\mathrm{K}_{2} \mathrm{TiF}_{6}$ [24]. Аппроксимация фотоэлектронных спектров для валентной зоны и остовных уровней была выполнена с помощью программы FitXPS [25].

Bсе измерения проводились при комнатной температуре и давлении остаточных газов в экспериментальной камере не более $2 \cdot 10^{-10}$ mbar. В процессе измерений заметных эффектов зарядки образцов, облучаемых интенсивным пучком монохроматизированного СИ мягкого рентгеновского диапазона, не наблюдалось.

\section{3. Результаты и обсуждение}

Порфирин никеля (NiP, $\mathrm{C}_{20} \mathrm{H}_{12} \mathrm{~N}_{4} \mathrm{Ni}$ ) представляет молекулярный комплекс, состоящий из центрального комплексообразующего атома никеля и устойчивого лиганда - порфинового макроцикла. Последний образован четырьмя пиррольными кольцами $\mathrm{NC}_{4}$, которые располагаются в одной плоскости и сцеплены между собой углеродными атомами $\mathrm{C}_{\alpha}$ через мостиковые атомы углерода $\mathrm{C}_{b}$ (рис. 1) [9]. Как следствие, комплекс $\mathrm{NiP}$ плоский и его атомное строение характеризуется группой $D_{4 h}$ точечной симметрии. Электронные свойства комплекса порфирина никеля определяются электронной структурой атома никеля и ковалентного порфинового макроцикла, а также их химическим взаимодействием. Ковалентное связывание атома никеля с макроциклом имеет донорно-акцепторный характер и осуществляется за счет смещения неподеленной $2 p$-электронной пары атома азота каждой из пиррольных групп на свободную $3 d$-орбиталь атома никеля [15]. В результате формируются четыре $s p^{2} d$-гибридизированные $\sigma$-связи, которые обеспечивают квадратную координацию атома никеля атомами азота.

Электронный спектр полиатомной системы (молекулы, комплекса) может быть описан с помощью спектрального распределения связывающих и разрыхляющих одноэлектронных МО этой системы, которые, как правило, характеризуют занятые и свободные электронные состояния полиатомной системы соответственно. $a$

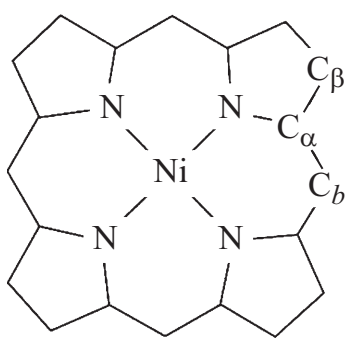

$b$

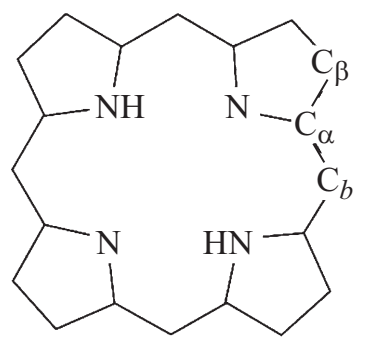

Рис. 1. Схематическое изображение молекул порфирина никеля $\mathrm{NiP}(a)$ и свободного порфина $\mathrm{H}_{2} \mathrm{P}(b)$.

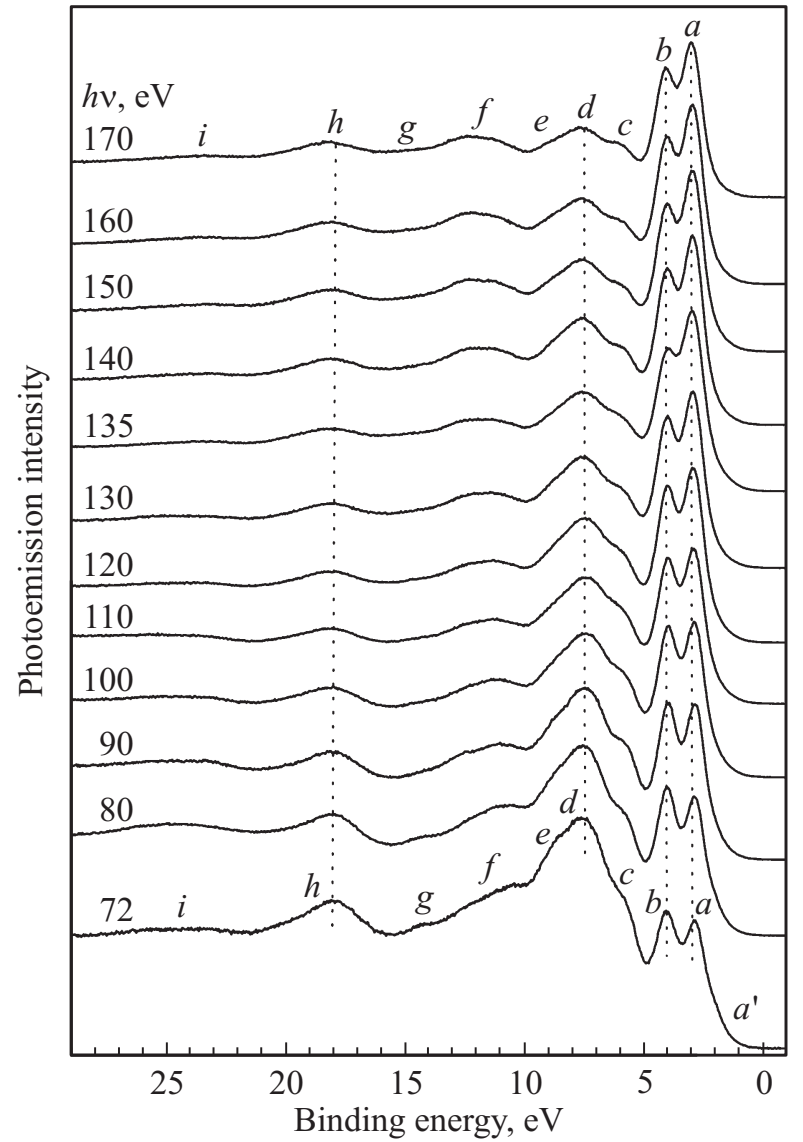

Рис. 2. Спектры валентной фотоэмиссии порфирина никеля $\mathrm{NiP}$, измеренные при разных энергиях возбуждающих квантов в диапазоне от 72 до $170 \mathrm{eV}$.

В случае плоской молекулы эти МО ориентированы различным образом относительно молекулярной плоскости: МО $\sigma$-типа располагаются в плоскости молекулы, а $\pi$-типа ориентированы перпендикулярно к ней [26]. Как правило, $\pi$-связывание более слабое в сравнении с $\sigma$-связыванием, что приводит к меньшим энергиям связи для связывающих и разрыхляющих МО $\pi$-типа.

В конденсированном состоянии $\mathrm{NiP}$ представляет собой молекулярный кристалл, в котором межмолекулярное сцепление характеризуется слабым ван-дерваальсовым взаимодействием [27]. Как следствие этого, электронная структура кристалла порфирина никеля в значительной степени определяется электронной структурой отдельной молекулы. Таким образом, валентная зона и зона проводимости кристалла NiP достаточно полно могут быть описаны отдельными узкими подзонами, которые происходят из одноэлектронных МО, описывающих занятые и свободные электронные состояния одной молекулы NiP.

Обсуждение полученных экспериментальных результатов начнем с рассмотрения спектров валентной фотоэмиссии порфирина никеля, измеренных при различных энергиях $h v$ возбуждающих квантов в диапазоне от 72 до $170 \mathrm{eV}$ (рис. 2). Фотоэмиссионные спектры, 
нормированные на интенсивность падающего излучения, представлены в шкале энергий связи валентных электронов относительно уровня Ферми. Интенсивность фотоэлектронного сигнала в области отрицательных энергий связи практически равняется нулю для каждого спектра.

Bсе спектры характеризуются двумя узкими линиями $a$ и $b$ вблизи уровня Ферми с энергиями связи 2.9 и $4.1 \mathrm{eV}$ соответственно, и более широкими ФЭ полосами $c-i$ при бо́льших энергиях. Кроме того, в спектрах, полученных при энергиях $h v$ возбуждающих квантов $72-110 \mathrm{eV}$, для линии $a$ наблюдается заметное плечо $a^{\prime}$, которое в других спектрах проявляется менее отчетливо. Следует отметить, что все ФЭ полосы сохраняют свои энергетические положения при изменении энергии возбуждающих квантов и, следовательно, обусловлены фотоионизацией верхних занятых электронных состояний, которые могут быть описаны занятыми одноэлектронными МО исследуемого комплекса.

Сравнивая спектры валентной фотоэмиссии порфирина никеля, замечаем, что при возрастании энергии $h v$ возбуждающих квантов интенсивности полос $c-i$ монотонно уменьшаются в несколько раз, примерно сохраняя свои относительные величины в спектрах. Наряду с этим, в рассматриваемом ряду спектров наблюдается значительное увеличение относительных интенсивностей линий $a$ и $b$ : они возрастают примерно в 4 раза относительно полосы $d$ (с фоном) при изменении энергии возбуждающих квантов от 72 до $170 \mathrm{eV}$. Кроме того, линия $a$ постепенно становится более интенсивной, чем линия $b$.

Обособленный характер полос $a$ и $b$ позволяет аппроксимировать спектры валентной ФЭ вблизи уровня Ферми $\left(E_{\text {bind }}=0-8 \mathrm{eV}\right)$ с помощью трех линий $a^{\prime}, a$ и $b$; результаты этой аппроксимации для трех спектров, измеренных с энергиями $h v$ возбуждающих квантов 72, 100 и $170 \mathrm{eV}$, показаны на рис. 3. Хорошо видно, что относительная интенсивность линии $a^{\prime}$ при энергии связи $2.0 \mathrm{eV}$ в сравнении с линией $a$ монотонно убывает от 0.26 до 0.05 при увеличении энергии квантов от 72 до $170 \mathrm{eV}$ (непоказанные на рисунке результаты аппроксимации для остальных спектров валентной фотоэмиссии согласуются с этим утверждением). Наряду с этим соотношение между интенсивностями пиков $b$ и $a$ меняется в пользу последнего. Таким образом, линия $a^{\prime}$ ведет себя при увеличении энергии $h v$ аналогично полосам $c-i$. Это наблюдение однозначно свидетельствует о близком атомно-орбитальном составе занятых МO, ответственных за полосы $c-i$ и $a^{\prime}$, и указывает на значительные различия между этими орбиталями и $\mathrm{MO}$, характеризующими занятые электронные состояния $a$ и $b$.

Действительно, вариации спектрального профиля валентной фотоэмиссии, наблюдаемые с ростом $h v$, отражают спектральные изменения сечений фотоионизации верхних занятых MO комплекса NiP. Эти сечения фотоионизации определяются, в свою очередь, соответствующими сечениями для валентных электронных оболочек атомов, составляющих молекулярный комплекс, т. е.

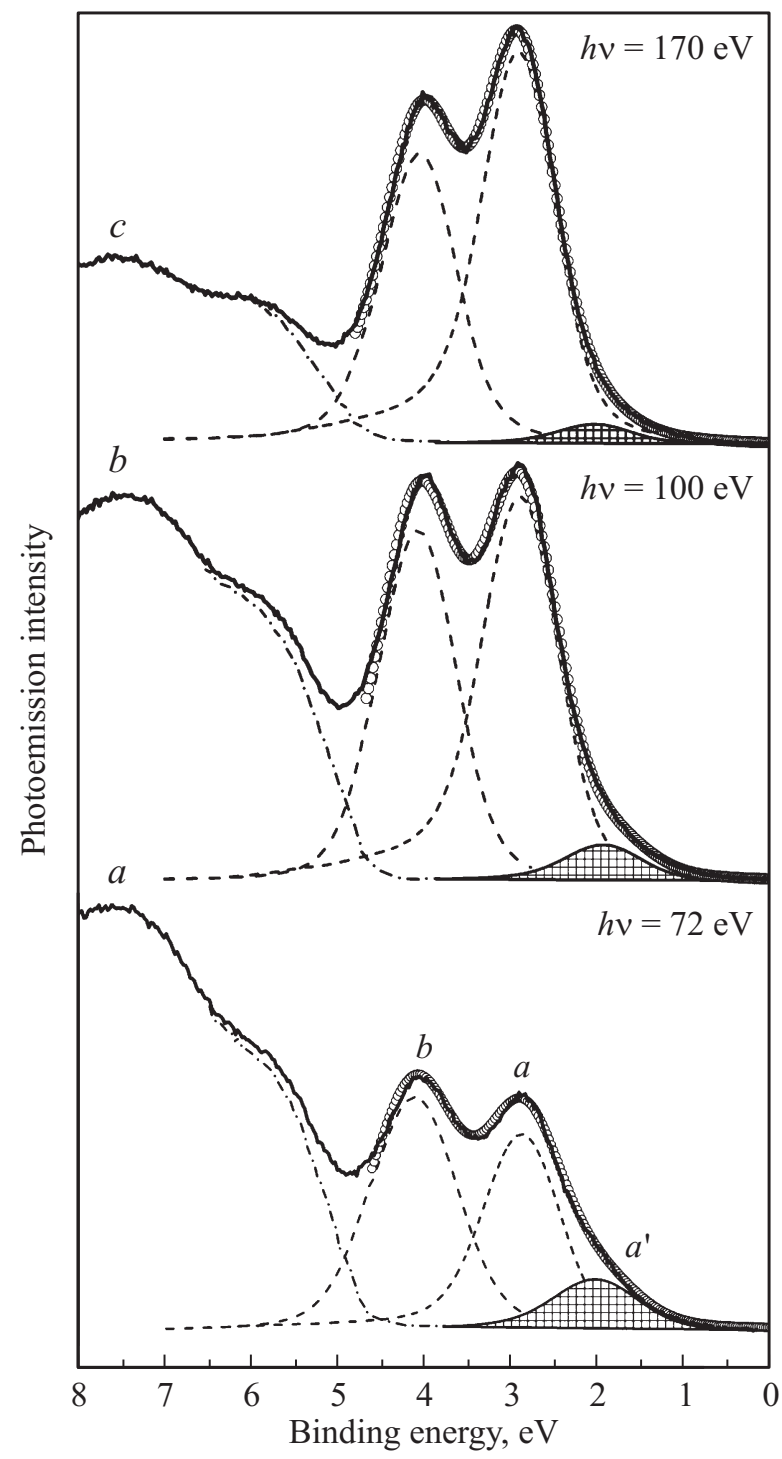

Рис. 3. Аппроксимация спектра валентной ФЭ порфирина никеля в области энергий связи $0-8 \mathrm{eV}$ с помощью линий $a^{\prime}$, $a$ и $b$ для спектров, полученных при энергиях квантов $72(a)$, $100(b)$ и 170 (c) eV. Экспериментальная кривая показана сплошной черной линией. Линии $a$ и $b$ в разложении изображены штриховыми линиями, контур линии $a^{\prime}$ заштрихован. Штрихпунктирной линией показан вклад близлежащих ФЭ полос $c$ и $d$ в анализируемую область спектра. Итоговый результат аппроксимации показан светлыми кружками

парциальными Ni3d-, N2p- и C2p-сечениями фотоионизации. Для оценки изменения этих величин обратимся к сечениям фотоионизации, рассчитанным для различных электронных оболочек атомов в диапазоне энергий квантов $h v$ от 0 до $1500 \mathrm{eV}$ [28]. Находим, что при $h v \sim 70 \mathrm{eV}$ сечения фотоионизации для $\mathrm{N} 2 p$ - и $\mathrm{C} 2 p$-оболочек составляют около 1.3 и $0.5 \mathrm{Mb}$ соответственно, тогда как для $\mathrm{Ni} 3 d$-электронной оболочки сечение равно примерно $8.5 \mathrm{Mb}$. При увеличении энергии квантов до $170 \mathrm{eV}$ все сравниваемые сечения монотонно убывают до $0.1 \mathrm{Mb}$ $(\mathrm{N} 2 p), 0.04 \mathrm{Mb}(\mathrm{C} 2 p)$ и $3 \mathrm{Mb}(\mathrm{Ni} 3 d)$. Другими словами, 
при возрастании энергии фотонов от 72 до $170 \mathrm{eV}$ сечения фотоионизации $\mathrm{N} 2 p$ - и $\mathrm{C} 2 p$-электронных оболочек убывают примерно в 13 раз, а $3 d$-сечение атома никеля убывает всего лишь в 3 раза, т.е. более чем в 4 раза медленнее $2 p$-сечений фотоионизации атомов азота и углерода.

Эти различия в скорости убывания рассчитанных сечений фотоионизации валентных электронных оболочек атома никеля, с одной стороны, и атомов углерода и азота - с другой, коррелируют с изменениями относительных интенсивностей линий $a$ и $b$ в сранении с полосами $a^{\prime}, c-i$ в экспериментальных спектрах при возрастании энергии возбуждающих квантов от 72 до $170 \mathrm{eV}$. Этот факт позволяет предположить, что ФЭ линии $a$ и $b$ связаны с МО порфирина никеля, которые сформированы в основном из $3 d$-орбиталей атома никеля, а полосы $a^{\prime}, c-i$ отражают МО порфинового макроцикла, в которых доминируют вклады валентных орбиталей атомов углерода и азота. Отметим, что наблюдаемая меньшая энергетическая ширина линий $a$ и $b$ в сравнении с полосами $c-i$ согласуется с высказанным соображением о связи этих ФЭ линий с занятыми $3 d$-состояниями атома никеля. Действительно, $3 d$-электроны сильно локализованы внутри атома никеля в результате коллапса электронной оболочки [29], и, как следствие, их состояния не могут сильно различаться (диспергировать) по энергии. Заметим также, что высокоэнергетические ФЭ полосы $h$ и $i$ с энергиями связи 18 и $24.5 \mathrm{eV}$, по всей видимости, связаны с $\mathrm{MO}$, образованными в основном из $\mathrm{C} 2 s-$ и $\mathrm{N} 2 s-\mathrm{AO}$, рассчитанные энергии связи которых в свободных атомах составляют 17.5 и $23.1 \mathrm{eV}$ соответственно [28].

Свободный атом никеля с электронной конфигурацией $[\operatorname{Ar}] 3 d^{8} 4 s^{2}$ в комплексе NiP выступает как двухвалентный атом $\mathrm{Ni}(\mathrm{II})$, который в рамках представлений о формальной валентности имеет электронную конфигурацию $[\mathrm{Ar}] 3 d^{8}$ иона $\mathrm{Ni}^{2+}$. В поле квадратного комплекса $\mathrm{NiP}$ (группа симметрии $D_{4 h}$ ) пятикратно вырожденные $3 d$-состояния иона никеля расщепляются (в порядке возрастания энергии) на одно двукратно вырожденное состояние $e_{g}\left(3 d_{x z, y z}\right)$ и три однократно вырожденных $a_{1 g}\left(3 d_{z^{2}}\right), b_{2 g}\left(3 d_{x y}\right)$ и $b_{1 g}\left(3 d_{x^{2}-y^{2}}\right)$ [30]. При этом три нижних $3 d$-состояния заполнены электронами $\left(e_{g}^{4}, a_{1 g}^{2}\right.$ и $b_{2 g}^{2}$ ), а энергетически наивысшее состояние $b_{1 g}$ остается свободным. Атомные $3 d$-орбитали никеля, описывающие эти состояния, участвуют наряду с $2 p$-орбиталями атомов азота порфинового лиганда в формировании МО $\sigma$ - и $\pi$-типа $\left(a_{1 g}, b_{1 g}\right.$ и $b_{2 g}, e_{g}$ соответственно), которые характеризуют химическое связывание между атомом никеля и лигандом в комплексе.

Учитывая предположение о связи ФЭ линий $a$ и $b$ с занятыми МО, имеющими большие вклады $\mathrm{Ni} 3 d$-состояний, логично считать ответственными за эти линии в спектре валентной фотоэмиссии три занятые $\mathrm{MO} e_{g}, a_{1 g}$ и $b_{2 g}$. Интенсивности линий $a$ и $b$ не сильно отличаются друг от друга, и, следовательно, эти линии связаны с МО, имеющими близкие по величине вклады
$\mathrm{Ni3} d$-состояний. Поэтому можно полагать, что линию а характеризуют два однократно вырожденных состояния $a_{1 g}$ и $b_{2 g}$, имеющих близкие энергии связи, а линию $b$ одно двукратно вырожденное состояние $e_{g}$.

Сравним выводы о характере верхних занятых МО в порфирине никеля с результатами расчетов, выполненных для этого комплекса в рамках теории функционала плотности (DFT) [9]. Прежде всего заметим, что порядок следования по энергии занятых МО с доминирующими $\mathrm{Ni} 3 d$-вкладами в DFT-расчетах $\left(b_{2 g}, e_{g}\right.$ и $\left.a_{1 g}\right)$ и теории кристаллического поля $\left(e_{g}, a_{1 g}\right.$ и $\left.b_{2 g}\right)$ не совпадает. Это указывает на важную роль эффектов химического связывания и процессов переноса электронного заряда между атомом никеля и порфиновым лигандом в формировании спектра занятых электронных состояний NiP.

Согласно расчетам, верхними заполненными МО в $\mathrm{NiP}$ являются высшая заполненная MO (highest occupied MO, НОМО) $a_{1 u}$ и следующая за ней НOMO-1 $a_{2 u}$, которые имеют энергии связи, совпадающие в пределах $0.01 \mathrm{eV}$. Эти MO локализованы на пиррольных кольцах порфинового лиганда и характеризуются доминирующими вкладами $2 p$-АО пиррольных атомов углерода $\left(\mathrm{C}_{\alpha}\right.$ и $\left.\mathrm{C}_{\beta}\right)$ для $\mathrm{HOMO}$ и $2 p-\mathrm{AO}$ атома азота и мостикового атома углерода $\mathrm{C}_{b}$ для НОМО-1. Этим МО, очевидно, соответствует $\Phi$ полоса $a^{\prime}$ в экспериментальном спектре (рис. 3).

Далее, при бо́льших энергиях связи, находятся согласно расчету две близко расположенные МО $a_{1 g}$ и $e_{g}$-симметрии, которые имеют доминирующие вклады $\mathrm{Ni} 3 d_{z^{2}}$ и $\mathrm{Ni} 3 d_{x z, y z}$-орбиталей. Принимая во внимание малость энергетического расстояния $\Delta E$ между этими $\mathrm{MO}(0.01 \mathrm{eV})$, логично полагать, что они ответственны за линию $a$ в спектре валентной фотоэмиссии. Более глубоко лежат еще две $\mathrm{MO} b_{2 g}$ - и $e_{g}$-симметрии с Ni3d-вкладами, практически совпадающие по энергии $(\Delta E=0.01 \mathrm{eV})$. Первая, $b_{2 g}-\mathrm{MO}$, является по существу чистой $3 d_{x y}$-орбиталью атома никеля, тогда как вторая в близких долях содержит вклады $\mathrm{Ni} 3 d_{x z, y z}, \mathrm{~N} 2 p-$ и $\mathrm{C}_{\beta} 2 p$-орбиталей. Это позволяет утверждать, что линия $b$ в основном характеризуется МО $b_{2 g}$-симметрии. Заметим, что в рамках данного расчета линии $a$ и $b$ связаны с другими компонентами $3 d-\mathrm{AO}$ атома никеля, чем предполагалось на основе анализа экспериментальных спектров валентной фотоэмиссии.

Кроме вышеупомянутых МО цитируемый расчет указывает на наличие в валентном спектре порфирина никеля еще четырех состояний, описываемых $\mathrm{MO} b_{2 u}$,

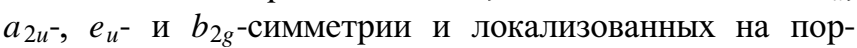
финовом макроцикле. Первые две МО располагаются энергетически между орбиталями, ответственными за фотоэлектронные линии $a$ и $b$, а вторые две имеют энергии связи немного больше, чем состояние $b$.

Результаты сравнения данных расчета с экспериментальным спектром валентной фотоэмиссии $\mathrm{NiP}$, к сожалению, позволяют рассматривать только их качественное согласие: высшее заполненное состояние локализовано на порфиновом макроцикле, ниже располагаются 
состояния с Ni3d-вкладами и далее снова идут состояния порфинового лиганда. Количественно сравниваемые данные согласуются не очень хорошо. Действительно, экспериментальный спектр валентных состояний NiP (полосы $a^{\prime}-g$, рис. 2) имеет энергетическую ширину около $12 \mathrm{eV}$, тогда как теоретический - всего лишь $4.3 \mathrm{eV}$. Для сравниваемых спектров это сопровождается также различиями в порядке следования МО, локализованных на атоме никеля и порфиновом макроцикле, и их взаимных энергетических положениях. Так, энергетическое расстояние между линиями $a^{\prime}$ и $a$ в экспериментальном спектре составляет $0.9 \mathrm{eV}$, а расстояние между соответствующими MO в расчете всего $0.3 \mathrm{eV}$. Между MO с Ni3d-вкладами в расчете располагаются МО порфинового лиганда, что представляется маловероятным по виду линий $a$ и $b$. Вместе с тем, необходимо отметить, что теоретическое энергетическое расщепление между двумя МO $\left(a_{1 g}+e_{g}\right.$ и $\left.b_{2 g}\right)$ с Ni3 $d$-вкладами $\mathrm{MO}$ составляет примерно $1.3 \mathrm{eV}$, что хорошо совпадает с экспериментальным энергетическим расстоянием $1.2 \mathrm{eV}$ между линиями $a$ и $b$.

Наконец, сравним относительные интенсивности экспериментальных линий $a$ и $b$ с их соотношением, ожидаемым из расчетов. Согласно последним интенсивность линии $a$ определяется $\mathrm{Ni3} d$-вкладами в $\mathrm{MO} a_{1 g^{-}}$и $e_{g}$-симметрии (0.97 и 0.71 соответственно), а линии $b-$ соответсвующим вкладом в $b_{2 g}$-МO $(0.93)$. Учитывая эти величины и двукратное вырождение МО $e_{g}$-симметрии, из расчета ожидаем соотношение 2.57:1 для интенсивностей линий $a$ и $b$. Сравнивая это соотношение с величинами, получаемыми из экспериментальных спектров - 0.84:1 $(h v=72 \mathrm{eV}), 1.08: 1(h v=100 \mathrm{eV})$ и $1.33: 1(h v=170 \mathrm{eV})$, приходим к выводу о плохом согласии между экспериментом и цитируемым расчетом.

Завершая сравнительный анализ экспериментальных данных и DFT-расчета [9] для верхних занятых электронных состояний в $\mathrm{NiP}$, следует заметить, что в расчетах $[9,31]$, выполненных одной группой авторов, приводится разный порядок следования верхних заполненных МО. Это расхождение объясняется различиями в выборе аппроксимации для обменного потенциала. O неоднозначности результатов DFT-расчетов для порфирина никеля указывают также данные работы [10], в которой рассчитанный порядок следования верхних заполненных MO отличается от порядка МO в вышеупомянутых работах.

Кратко рассмотрим теперь рентгеновские фотоэлектронные спектры (XPS) остовных Ni2p-, N1s- и $\mathrm{C} 1 s$-электронов (рис. 4), которые содержат информацию о химическом состоянии атомов в $\mathrm{NiP}$ и необходимы для энергетического совмещения спектров поглощения разных атомов на единой энергетической шкале. Полученные XPS-спектры представлены на шкале энергий связи, измеренных относительно уровня Ферми. Ni2 $p$-спектр состоит из двух спин-дублетных компонентов - Ni2 $p_{1 / 2}$ и $\mathrm{Ni} 2 p_{3 / 2}$, расположенных при энергиях связи 873.0 и $855.7 \mathrm{eV}$ соответственно. Эти величины существен-

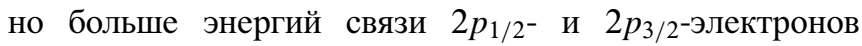

относительно уровня Ферми в металлическом никеле $(870.0$ и $852.7 \mathrm{eV})$ [32], что указывает на значительный перенос электронной плотности с атома никеля на порфиновый лиганд в $\mathrm{NiP} . \mathrm{B} \mathrm{Ni} 2 p_{3 / 2}$-спектре при энергии связи $863.5 \mathrm{eV}$ отчетливо наблюдается высокоэнергетический сателлит $s h$, свидетельствующий о заметной роли многоэлектронных (shake-off) эффектов в процессе фотоионизации электронной $2 p_{3 / 2}$-оболочки атома никеля [33]. Фотоэлектронный $\mathrm{N} 1 s$-спектр также характеризуется одиночной линией простой формы при энергии связи $399.15 \mathrm{eV}$, что указывает на одинаковое химическое состояние всех атомов азота в комплексе. Слабый высокоэнергетический сателлит $s h$ при энергии $402.3 \mathrm{eV}$ отражает наличие shake-off процессов при фотоионизации N1s-оболочки [34].

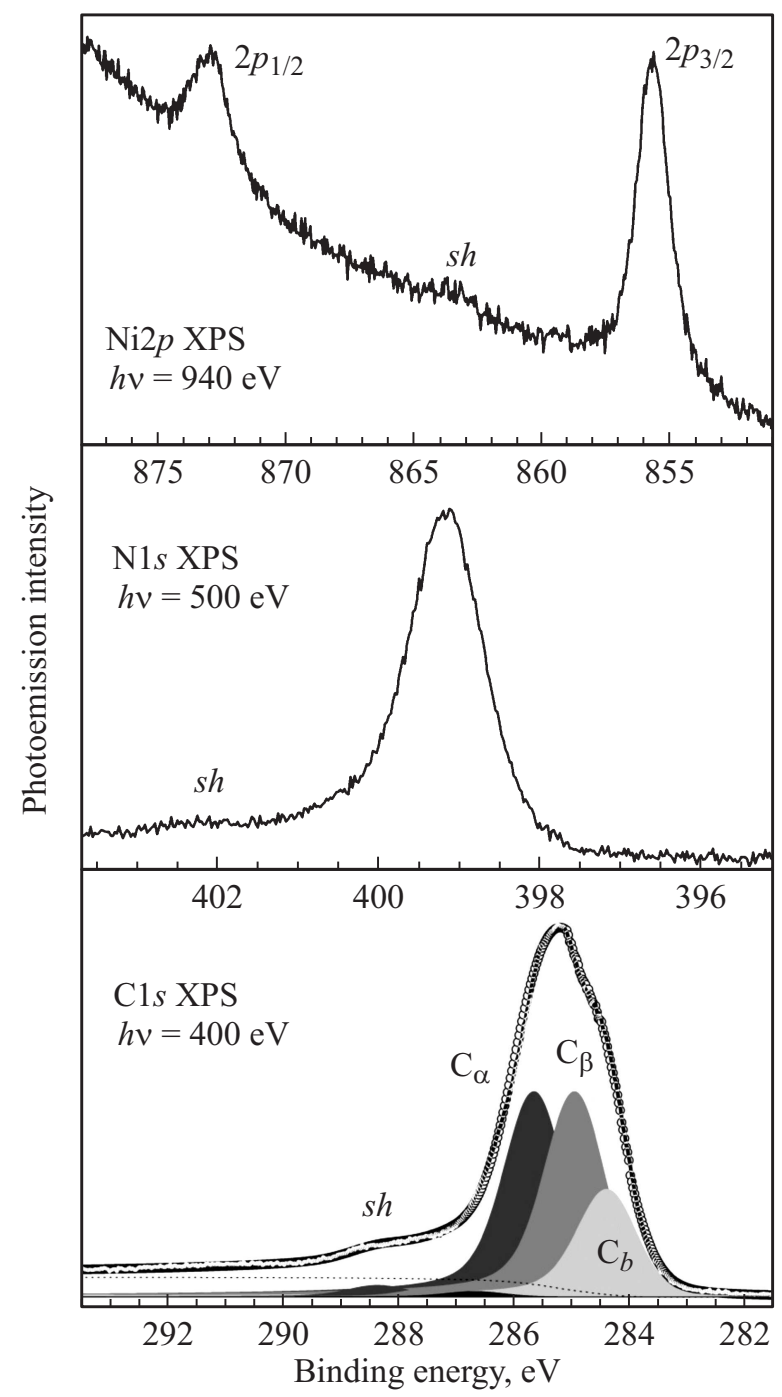

Рис. 4. Ni2p-, N1s- и C1s-фотоэлектронные спектры порфирина никеля NiP. Аппроксимация для $\mathrm{C} 1 s$-спектра: экспериментальная кривая показана сплошной линией; контуры фотоэлектронных линий, соответствующих атомам углерода в разных химических состояниях $\left(\mathrm{C}_{b}, \mathrm{C}_{\alpha}, \mathrm{C}_{\beta}\right)$, закрашены; штриховой линией изображен фон; итоговый спектр показан светлыми кружками. 
В отличие от спектров никеля и азота $\mathrm{C} 1 s$-спектр имеет более сложный асимметричный профиль, который удается аппроксимировать тремя компонентами $\mathrm{C}_{\alpha}$, $\mathrm{C}_{\beta}$ и $\mathrm{C}_{b}$, которые соответствуют трем типам атомов углерода, находящимся в $\mathrm{NiP}$ в разных химических состояниях (рис. 1). Относительные интенсивности этих компонентов отражают количество разных атомов углерода в комплексе $\mathrm{C}_{\alpha}: \mathrm{C}_{\beta}: \mathrm{C}_{b}=2: 2: 1$, а их энергии $285.65 \mathrm{eV}\left(\mathrm{C}_{\alpha}\right), 284.95 \mathrm{eV}\left(\mathrm{C}_{\beta}\right)$ и $284.38 \mathrm{eV}\left(\mathrm{C}_{b}\right)$ - отражают химическое (зарядовое) состояние этих атомов углерода в порфирине никеля. Заметные различия энергий $\mathrm{C} 1 s$-электронов характеризуют величины и направления электронного переноса между атомами углерода, обусловленного наличием в пиррольном кольце более электроотрицательного гетероатома азота.

Для получения информации о свободных электронных состояниях в NiP и их свойствах рассмотрим ближнюю тонкую структуру рентгеновских спектров поглощения (NEXAFS) комплекса в области Ni2p-, $\mathrm{N} 1 s$ - и $\mathrm{C} 1 s$-порогов ионизации (рис. 5). Обозначения абсорбционных полос в спектрах даны с учетом результатов их последующей идентификации. Измеренный $2 p$-спектр поглощения атома никеля состоит из $2 p_{3 / 2^{-}}$и $2 p_{1 / 2}$-компонентов, обусловленных спинорбитальным расщеплением начального Ni2p-состояния $(17.3 \mathrm{eV}$ [32]). Тонкая структура этих спектров формируется в основном дипольно-разрешенными переходами

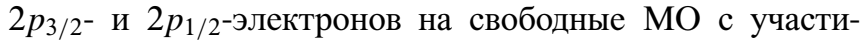
ем $3 d$-состояний атома никеля, поскольку разрешенные переходы $2 p$-электронов в $4 s$-состояния примерно в 20 раз менее интенсивные по сравнению с переходами в $3 d$-состояния [35]. В дальнейшем мы ограничимся рассмотрением только низкоэнергетического $2 p_{3 / 2}$-компонента, имеющего более четкую и интенсивную структуру. В $2 p_{1 / 2}$-спектре структура разрешена существенно хуже, поскольку начальный $\mathrm{Ni} 2 p_{1 / 2}$-уровень имеет в сравнении с $\mathrm{Ni} 2 p_{3 / 2}$-уровнем заметно бо́льшую собственную ширину вследствие существования дополнительного Оже- $2 p_{1 / 2} 2 p_{3 / 2} 3 d$-процесса распада для $\mathrm{Ni} 2 p_{1 / 2}$-вакансии [36].

Для $\mathrm{C} 1 s$ - и $\mathrm{N} 1 s$-спектров поглощения $\mathrm{NiP}$ и $\mathrm{H}_{2} \mathrm{P}$ характерно наличие двух областей с различными абсорбционными структурами - низкоэнергетической области с узкими линиями и высокоэнергетической области с широкими полосами поглощения. Линии $B-C$ в $\mathrm{N} 1 s$-спектрах $\left(B-B_{2}\right.$ в $\mathrm{C} 1 s$-спектрах $) \mathrm{NiP}$ и $\mathrm{H}_{2} \mathrm{P}$ заметно различаются по свой форме и энергетическому положению, тогда как высокоэнергетические абсорбционные полосы $D-F$ в спектрах азота и $C-F$ в спектрах углерода практически совпадают по форме и энергетическим положениям в сравниваемых спектрах.

Ближнюю тонкую структуру рентгеновских спектров поглощения принято описывать с помощью многократного (резонансного) рассеяния фотоэлектронов, вырываемых из атома в результате поглощения рентгеновского кванта, на атомах ближайшего окружения [13,24]. При определенных энергиях фотоэлектрона квазимолекула,

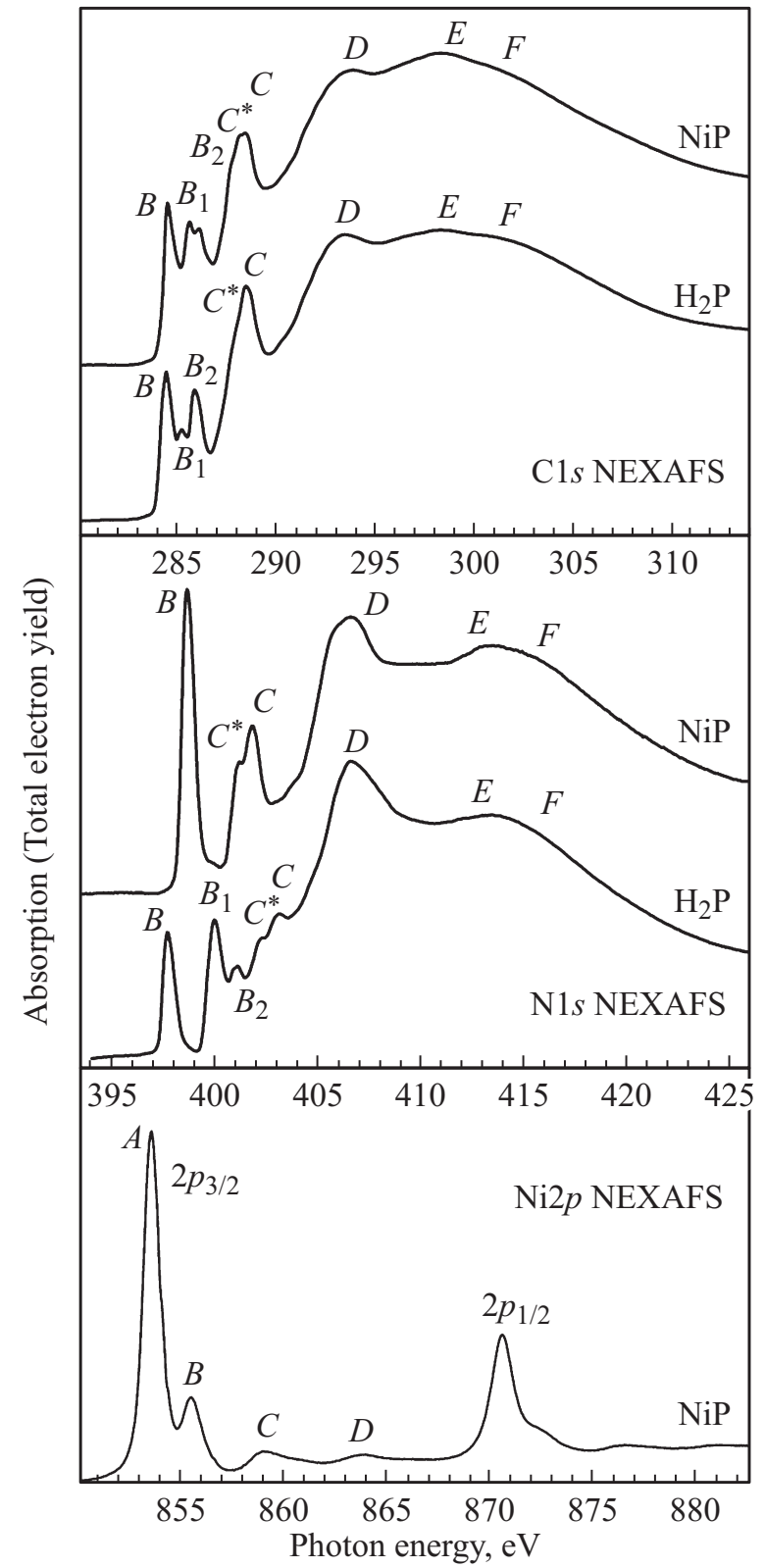

Рис. 5. Ni2 $2 p_{3 / 2^{-},} \mathrm{N} 1 s-$ и $\mathrm{C} 1 s$-спектры поглощения порфирина никеля NiP. Для сравнения приведены $\mathrm{N} 1 s$ - и $\mathrm{C} 1 s$-спектры поглощения свободного порфина $\mathrm{H}_{2} \mathrm{P}$.

образуемая атомами окружения, может временно захватывать фотоэлектрон, приводя к образованию для него метастабильных состояний (резонансов формы). Эти резонансы в зависимости от их времени жизни наблюдаются в спектрах в виде узких линий или широких полос поглощения. Локализация резонансов в поле квазимолекулы позволяет рассматривать их как результат дипольно-разрешенных переходов остовных электронов на свободные электронные состояния этой полиатомной системы, которые описываются ее незанятыми МO.

В рамках такого подхода к описанию NEXAFSспектров логично утверждать, что отличия в низкоэнергетической части NEXAFS-спектров $\mathrm{NiP}$ и $\mathrm{H}_{2} \mathrm{P}$ отра- 


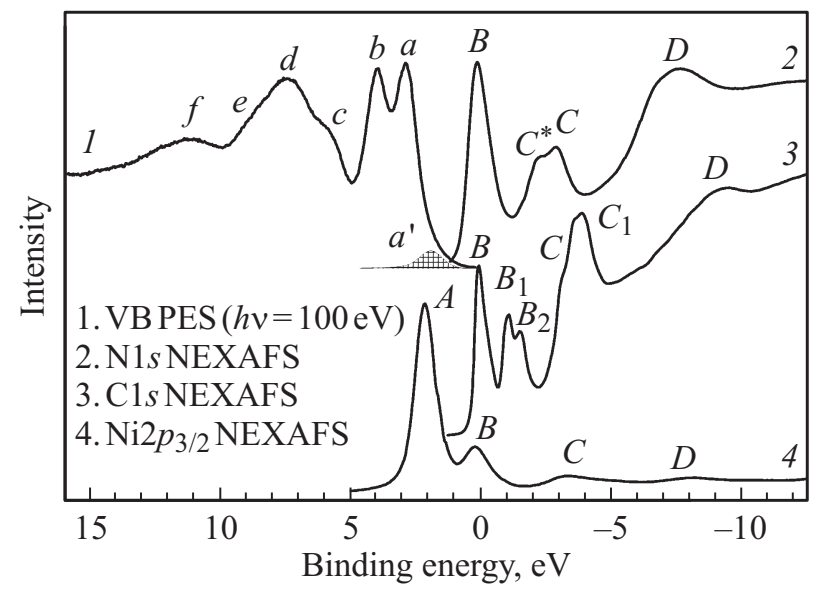

Рис. 6. Спектр валентной фотоэмиссии VB PES $(h v=100 \mathrm{eV})$ и $\mathrm{Ni} 2 p_{3 / 2^{-}}, \mathrm{N} 1 s-$ и $\mathrm{C} 1 s$-спектры поглощения порфирина никеля $\mathrm{NiP}$, приведенные к единой энергетической шкале энергий связи относительно уровня Ферми.

жают различия в их локальной электронной структуре, которые обусловлены изменением центральных атомов комплексов и характера их химического связывания с соседними атомами азота порфинового лиганда в $\mathrm{NiP}$ в сравнении с $\mathrm{H}_{2} \mathrm{P}$. В свою очередь высокоэнергетические полосы поглощения в спектрах обоих комплексов связаны с процессами формирования тонкой структуры в пределах жесткого порфинового лиганда, который мало меняется при переходе от $\mathrm{NiP}$ к $\mathrm{H}_{2}$ Р. Из выполненного сравнения очевидно, что информация об особенностях локальной электронной структуры комплекса, обусловленных химическим связыванием атома никеля с порфиновым лигандом, содержится в основном в низкоэнергетической области спектров поглощения.

Рассмотрим информативную область спектров поглощения NiP в рамках квазимолекулярного подхода, полагая, что локальная электронная структура комплекса в значительной степени определяется полиатомной группой (квазимолекулой) $\mathrm{NiN}_{4}\left(\mathrm{C}_{\alpha}\right)_{8}$, которую образует центральный комплексообразующий атом никеля с атомами азота и углерода первой и второй координационных сфер его окружения (рис. 1). Эта квазимолекула, как и молекула порфирина никеля в целом, характеризуется группой точечной симметрии $D_{4 h}$. При таком подходе тонкая структура сравниваемых спектров поглощения изучаемой полиатомной системы может рассматриваться единообразно как результат дипольноразрешенных переходов остовных электронов на общую систему незанятых электронных состояний комплекса, которые могут быть аппроксимированы свободными МО квазимолекулы [24].

На рис. 6 низкоэнергетическая часть спектров поглощения NiP представлена вместе со спектром валентной фотоэмиссии $(h v=100 \mathrm{eV})$ на единой шкале энергий связи, определенной относительно уровня Ферми. Спектры поглощения приведены к данной шкале, используя рассмотренные выше энергии связи для $\mathrm{Ni} 2 p_{3 / 2}, \mathrm{~N} 1 s$ и $\mathrm{C}_{\alpha} 1 s$-электронов - 855.7, 399.15 и $285.65 \mathrm{eV}$ соответственно. Заметим, что в случае спектра углерода была взята энергия связи атомов $\mathrm{C}_{\alpha}$, непосредственно взаимодействующих с атомами азота в комплексе и входящих в состав используемой квазимолекулы. Рассмотрение тонкой структуры спектров поглощения, отнесенных к единой энергетической шкале, в рамках квазимолекулярного подхода предполагает совпадение в сравниваемых спектрах энергетических положений абсорбционных линий и полос, связанных с переходами на одни и те же МО.

Из энергетически совмещенных спектров NiP прежде всего замечаем, что абсорбционный резонанс $A$, соответствующий низшему свободному состоянию комплекса, наблюдается только в Ni2p $3 / 2$-спектре поглощения. Отсюда непосредственно следует, что это состояние характеризуется низшей незанятой MO (lowest unoccupied MO, LUMO) $b_{1 g}$-симметрии с вкладом $3 d_{x^{2}-y^{2}}$-состояний атома никеля, которые остаются незанятыми для двухвалентного атома $\mathrm{Ni}(\mathrm{II})$ в молекулярном поле квадратного $\left(D_{4 h}\right)$ комплекса. Согласно расчетам [9, 10] LUMO в NiP является слабо разрыхляющей $b_{1 g}$-MO $\sigma$-типа, в которую наряду с $\mathrm{Ni}$ $3 d_{x^{2}-y^{2}}$-АО заметный вклад вносят $2 p$-состояния атомов азота. Однако в $\mathrm{N} 1 s$-спектре поглощения отсутствует даже намек на какую-либо структуру, подобную полосе $A$ в $\mathrm{Ni} 2 p_{3 / 2}$-спектре. В работе [20] для объяснения этого наблюдения было предположено, что реальный вклад $\mathrm{N} 2 p$-состояний в LUMO значительно меньше расчетной величины $(\sim 0.33)[10,31]$. Вместе с тем следует отметить, что по своему энергетическому положению пик $A$ точно попадает на полосу $a^{\prime}$ в спектре валентной фотоэмиссии, которая отражает высшее заполненное состояние комплекса, связанное с порфиновым лигандом. Ранее она была приписана практически совпадающим по энергии НОМО и НОМО-1 $a_{1 u^{-}}$и $a_{2 u^{-}}$-симметрии. На первый взгляд такая корреляция абсорбционного пика $A$ и фотоэмиссионной полосы $a^{\prime}$ представляется маловероятной.

Однако донорно-акцепторный механизм формирования химической $\sigma$-связи в $\mathrm{NiP}$ предполагает участие в связывании неподеленных $2 p$-электронных пар четырех атомов азота. Каждая пара смещается в область свободного $\mathrm{Ni} 3 d_{x^{2}-y^{2}}$-состояния и формирует двухэлектронную ковалентную связь между атомом никеля и атомом азота, которая радикально отличается от обычной двухцентровой ковалентной связи [11]. Согласно DFTрасчету [9], НОМО-1 $a_{2 u}$-симметрии имеет значительный вклад $\mathrm{N} 2 p$-состояний, который, по всей видимости, и представляет неподеленную электронную пару атома азота. Так как фотоэмиссионная полоса $a^{\prime}$ связана с HOMO-1, то становится понятной ее энергетическая корреляция с резонансом $A$ в $\mathrm{Ni} 2 p_{3 / 2}$-спектре поглощения. Поэтому логично утверждать, что отсутствие аналога полосы $A$ в спектре азота обусловлено специфическим составом LUMO, в формировании которой 
незанятые Ni3d-состояния участвуют наряду с занятыми $2 p$-состояниями неподеленных электронных пар атомов азота. В этом случае переходы N1s-электронов в LUMO просто невозможны, поскольку N2p-состояния в ней заняты.

Следующий абсорбционный резонанс $B$ отчетливо наблюдается во всех сравниваемых спектрах поглощения $\mathrm{NiP,} \mathrm{причем} \mathrm{его} \mathrm{энергетическое} \mathrm{положение} \mathrm{в} \mathrm{разных}$ спектрах совпадает в пределах точности эксперимента $(\sim 0.1 \mathrm{eV})$. Из этого однозначно следует, что следующее свободное состояние $\mathrm{LUMO}+1$, которое ответственно за резонанс $B$, представляет собой молекулярное состояние (MO) с вкладами $\mathrm{Ni3d-}, \mathrm{N} 2 p$ - и $\mathrm{C}_{\alpha} 2 p$-состояний. Гибридизированный характер $\mathrm{LUMO}+1$ подтверждается расчетом [9], согласно которому это свободное состояние приписывается $e_{g}$-MO $\pi$-типа с небольшим вкладом Ni3 $d_{x z, y z} \pi$-состояний и значительными вкладами $2 p \pi$-состояний атомов порфинового лиганда. Рассчитанные характеристики второго свободного состояния в $\mathrm{NiP}$ качественно согласуются с экспериментальными результатами. Действительно, пики $B$ в спектрах азота (2) и углерода (3) по своей форме соответствуют переходам $1 s$-электронов в свободные $\pi$-состояния молекулярного комплекса, а их большие интенсивности в сравнении со спектром никеля отражают тот факт, что $\mathrm{N} 2 p$ - и $\mathrm{C} 2 p$-вклады в LUMO +1 в несколько раз превышают вклад Ni3d-состояний [9].

Как уже отмечалось, $3 d$-электроны сильно локализованы во внутриатомной области атома никеля благодаря коллапсу их электронной оболочки, что находит свое отражение в небольшой ширине фотоэлектронных линий $a$ и $b$ в спектре валентной фотоэмиссии $\mathrm{NiP}$ (рис. 2). Вместе с тем значительная интенсивность полосы $B$ в спектре поглощения никеля указывает на то, что в этом комплексе имеет место частичная делокализация $3 d$-электронов. В работах $[19,20]$ подобная делокализация в порфиринах и фталоцианине никеля была связана с сильным $\pi$-связыванием между атомами никеля и лиганда. Это связывание осуществляется за счет ковалентного $\mathrm{Ni} 3 d_{x z, y z}-\mathrm{N}, \mathrm{C} 2 p$ смешивания и сопровождается зарядовым переносом Ni3d-электронной плотности на атомы лиганда (эффект обратного донирования [26]). Наличие $\pi$-связывания между атомами никеля и лиганда в порфирине никеля подтверждается всеми DFT-расчетами, однако рассчитанная величина переноса $\mathrm{Ni} 3 d$-электронной плотности (вклада в LUMO + 1) невелика ( 0.03) [9] и не позволяет объяснить значительную интенсивность полосы $B$ в Ni2 $p_{3 / 2}$-спектре, которая составляет примерно одну четверть от интенсивности основного перехода (резонанса $A$ ) на LUMO $b_{1 g}$-симметрии с вкладом $\mathrm{Ni3} d$-состояний, равным 0.59. Далее, расчет не согласуется с экспериментом и при сравнении энергетического расстояния LUMO и LUMO $+1, \Delta E\left(b_{1 g}-e_{g}\right)$, которое равно $0.35 \mathrm{eV}$ в расчете [9] и $2.1 \mathrm{eV}$ в эксперименте.

Пики $B_{1}$ и $B_{2}$ наблюдаются только в $\mathrm{C} 1 s$-спектре поглощения $\mathrm{NiP}$, что указывает на их связь со свободными МО порфинового лиганда. Энергетическое расстояние между ними, равное $0.47 \mathrm{eV}$, в пределах экспериментальной точности $(\sim 0.1 \mathrm{eV})$ совпадает с расстоянием $0.55 \mathrm{eV}$ между $\mathrm{C}_{\beta} 1 s-$ и $\mathrm{C}_{b} 1 s$-уровнями в порфирине никеля. Это позволяет предположить, что резонансы $B_{1}$ и $B_{2}$ в спектре поглощения углерода отражают переходы $1 s$-электронов атомов углерода $\mathrm{C}_{\beta}$ и $\mathrm{C}_{b}$, непосредственно не связанных с атомами никеля и азота, в одно и то же свободное состояние порфинового лиганда. Вполне возможно, что этому состоянию соответствует расчетная $\mathrm{LUMO}+2 b_{1 u}$-симметрии, которая в основном локализована на атомах углерода $C_{\beta}$ и $C_{b}$ и не имеет вкладов от атомов никеля и азота.

Далее, малоинтенсивным полосам $C$ и $D$ в $\mathrm{Ni} 2 p_{3 / 2}$ спектре в принципе можно указать аналоги в $1 s$-спектрах азота и углерода, однако идентификация этих полос на данном этапе затруднительна и можно только предположить, что они связаны с переходами остовных электронов на разрыхляющие МО, имеющие вклады $\mathrm{Ni} 4 s, 4 p$ - и $\mathrm{N}, \mathrm{C} 2 p$-состояний.

\section{4. Заключение}

Энергетические распределения и свойства занятых и свободных электронных состояний для плоского комплекса порфирина никеля $\mathrm{NiP}$ исследованы методами рентгеновской фотоэмиссионной и абсорбционной спектроскопии.

Закономерности в поведении спектра валентной фотоэмиссии при увеличении энергии возбуждающих квантов от 72 до $170 \mathrm{eV}$ проанализированы на основе теоретических зависимостей спектрального поведения парциальных $\mathrm{Ni} 3 d$-, $\mathrm{N} 2 p$ - и $\mathrm{C} 2 p$-сечений фотоионизации. В результате установлено, что высшее заполненное состояние комплекса порфирина никеля с энергией связи $2.0 \mathrm{eV}$ характеризуется MO, построенной из атомных состояний порфинового лиганда. Немного глубже при энергиях 2.9 и $4.1 \mathrm{eV}$ располагаются занятые $\mathrm{MO}$ комплекса, которые сформированы главным образом из $\mathrm{Ni} 3 d$-состояний. В диапазоне энергий связи от 5 до $15 \mathrm{eV}$ находятся занятые МО порфинового лиганда.

Фотоэлектронные $\mathrm{Ni} 2 p_{3 / 2^{-}}$и $\mathrm{N} 1 s$-спектры характеризуются одиночными линиями простой формы, тогда как $\mathrm{C} 1 s$-спектр имеет более сложный асимметричный профиль. Последний удается аппроксимировать тремя компонентами $\mathrm{C}_{\alpha}, \mathrm{C}_{\beta}$ и $\mathrm{C}_{b}$, которые соответствуют трем типам атомов углерода, находящимся в NiP в разных химических состояниях.

Сравнительный анализ $\mathrm{C} 1 s-$ и $\mathrm{N} 1 s$-спектров поглощения NiP со спектрами свободного порфина $\mathrm{H}_{2} \mathrm{P}$ показал, что замещение атомов водорода на атом никеля не оказывает существенного влияния на порфиновый лиганд, но влияет на химическое состояние атомов азота и углерода $\mathrm{C}_{\alpha}$, что приводит к изменениям в начальной области тонкой структуры $\mathrm{N} 1 s-$ и $\mathrm{C} 1 s$-спектров.

Тонкая структура спектров поглощения и спектр валентной фотоэмиссии NiP рассмотрены в рамках квазимолекулярного подхода на единой энергетической 
шкале энергий связи (относительно уровня Ферми). В результате установлено, что низшее свободное состояние LUMO имеет специфический характер и описывается МО $\sigma$-типа, которая формируется свободными $\mathrm{Ni} 3 d_{x^{2}-y^{2}}$-состояниями и занятыми $2 p$-состояниями неподеленных электронных пар атомов азота. В этом случае переходы N1 $s$-электронов в LUMO невозможны, поскольку N2p-состояния в ней заняты. Этот специфический характер LUMO является следствием донорноакцепторной химической связи в NiP.

Следующее незанятое состояние LUMO + 1 приписывается $e_{g}$-MO $\pi$-типа с небольшим вкладом $\mathrm{Ni} 3 d_{x z, y z} \pi$ состояний и значительными вкладами $2 p \pi$-состояний атомов порфинового лиганда. Это $\pi$-связывание осуществляется за счет ковалентного $\mathrm{Ni} 3 d_{x z, y z}-\mathrm{N}$, $\mathrm{C} 2 p$-смешивания и сопровождается зарядовым переноcom Ni3d-электронной плотности на атомы лиганда (эффект обратного допирования).

В результате непосредственного сравнения установлено, что экспериментальные спектры валентной фотоэмиссии и поглощения согласуются с результатами DFT-расчетов только на качественном уровне.

Авторы выражают благодарность М.М. Бржезинской (БЭССИ) за помощь при проведении измерений.

\section{Список литературы}

[1] The Porphyrin Handbook / Eds K.M. Kadish, K.M Smith, R. Guilard. V. 1-10. CA: Academic, San Diego (2000).

[2] H.L. Anderson. Chem. Commun., 2323 (1999).

[3] O. Senge, M. Fazekas, E.G.A. Notaras, W.J. Blau, M. Zawadzka, O.B. Locos, E.M. Ni Mhuiercheartaigh. Adv. Mater. 19, 2737 (2007).

[4] P. Bhyrappa, J.K. Young, J.S. Moore, K.S. Suslick. J. Am. Chem. Soc. 118, 5708 (1996).

[5] Manivannan Ethirajan, Yihui Chen, Penny Joshi, Ravindra K. Pandey. Chem. Soc. Rev. 40, 340 (2011).

[6] D. Filippini, A. Alimelli, C. Di Natale, R. Paolesse, A. D’Amico, I. Lundström. Angew. Chem. Int. Ed. 45, 3800 (2006).

[7] M. Gouterman. J. Mol. Spectr. 6, 138 (1961).

[8] A. Antipas, M. Gouterman. J. Am. Chem. Soc. 105, 4869 (1983).

[9] A. Rosa, G. Ricciardi, E.J. Baerends, S.J.A. van Gisbergen. J. Phys. Chem. A 105, 3311 (2001).

[10] M.S. Liao, S. Scheiner. Chem. Phys. 117, 205 (2002).

[11] J.S. Evans, R.L. Musselman. Inorg. Chem. 43, 5613 (2004).

[12] D. Kim, C. Kirmaier, D. Holten. Chem. Phys. 75, 305 (1983).

[13] J. Stöhr. NEXAFS Spectroscopy. Springer Series in Surface Science. Springer Verlag, Berlin (1992). V. 25. 403 p.

[14] S. Hüfner. Photoelectron Spectroscopy. Principles and Applications. Second Ed. Springer, Berlin (1995). 507 p.

[15] C. Berrios, G.I. Cárdenas-Jirón, J.F. Marco, C.Gutiérrez, M.S. Ureta-Zañartu. J. Phys. Chem. A 111, 2706 (2007).

[16] C. Berrios, J.F. Marco, C.C. Gutiérrez, M.S. Ureta-Zañartu. J. Phys. Chem. B 112, 12644 (2008).

[17] K.M. Barkigia, M.W. Renner, L.R. Furenlid, C.J. Medforth, K.M. Smith, J. Fajer. J. Am. Chem. Soc. 115, 3627 (1993).
[18] L. Campbell, S. Tanaka, S. Mukamel. Chem. Phys. 299, 225 (2004).

[19] S.A. Krasnikov, A.B. Preobrajenski, N.N. Sergeeva, M.M. Brzhezinskaya, M.A. Nesterov, A.A. Cafolla, M.O. Senge, A.S. Vinogradov. Chem. Phys. 332, 318 (2007).

[20] S.A. Krasnikov, N.N. Sergeeva, M.M. Brzhezinskaya, A.B. Preobrajenski, Y.N. Sergeeva, N.A. Vinogradov, A.A. Cafolla, M.O. Senge, A.S. Vinogradov. J. Phys.: Condens. Matter 20, 235207 (2008).

[21] S.I. Fedoseenko, I.E. Iossifov, S.A. Gorovikov, J.-S. Schmid, R. Follath, S.L. Molodtsov, V.K. Adamchuk, G. Kaindl. Nucl. Instrum. Meth. Phys. Res. A 470, 84 (2001).

[22] R. Nyholm, S. Svensson, J. Nordgren, A. Flodström. Nucl. Instrum. Methods A 246, 267 (1986).

[23] E. Unger, U. Bobinger, W. Dreybrodt, R. Schweitzer-Stenner. J. Phys. Chem. 97, 9956 (1993).

[24] A.S. Vinogradov, S.I. Fedoseenko, S.A. Krasnikov, A.B. Preobrajenski, V.N. Sivkov, D.V. Vyalikh, S.L. Molodtsov, V.K. Adamchuk, C. Laubschat. G. Kaindl. Phys. Rev. B 71, 045127 (2005).

[25] Adams, D.L. FitXPS, v. 2.12, http://ww2.sljus.lu.se/download.html

[26] Ф. Коттон, Дж. Уилкинсон. Основы неорганической химии. Мир, М. (1979). 680 с.

[27] S.R. Forrest. Chem. Rev. 97, 1793 (1997).

[28] J.J. Yeh, I. Lindau. Atomic Data and Nuclear Data Tables 32, 1 (1985).

[29] Р.И. Каразия. Успехи физ. наук 135, 79 (1981).

[30] И.Б. Берсукер. Электронное строение и свойства координационных соединений. Введение в теорию. 3-е изд. Химия, Л. (1986). 288 с.

[31] A. Rosa, G. Ricciardi, E.J. Baerends, M. Zimin, M.A.J. Rodgers, S. Matsumoto, N. Ono. Inorg. Chem. 44, 6609 (2005).

[32] J.C. Fuggle, N. Mårtensson. J. Electron Spectrosc. Relat. Phenom. 21, 275 (1980).

[33] M.C. Biesinger, B.P. Payne, A.P. Grosvenor, L.W.M. Lau, A.R. Gerson, R.St.C. Smart. Appl. Surf. Sci. 257, 2717 (2011).

[34] M.P. Keane, A. Naves de Brito, N. Correia, S. Svensson. Chem. Phys. 155, 379 (1991).

[35] H. Ebert, J. Stöhr, S.S.P. Parkin, M. Samant, A. Nilsson. Phys. Rev. B 53, 16067 (1996).

[36] J.G. Chen. Surf. Sci. Rep. 30, 1 (1997). 